\title{
Trajectory Tracking Control of a Hydraulic System Using TSMCSPO based on Sliding Perturbation Observer
}

\author{
Jie Wang ${ }^{1} \oplus$, Min Cheol Lee ${ }^{1, *}$, Karam Dad Kallu ${ }^{1}$, Saad Jamshed Abbasi ${ }^{1}$ and \\ Seokyoung Ahn 2 (D) \\ 1 Measurement and Control Laboratory, School of Mechanical Engineering, Pusan National University, \\ Busan 46241, Korea; wj@pusan.ac.kr (J.W.); karamdadkallu@gmail.com (K.D.K.); \\ saadjamshed93@gmail.com (S.J.A.) \\ 2 Advanced Material Processing Laboratory, School of Mechanical Engineering, Pusan National University, \\ Busan 46241, Korea; sahn@pusan.ac.kr \\ * Correspondence: mclee@pusan.ac.kr; Tel.: +82-051-510-3081
}

Received: 14 March 2019; Accepted: 3 April 2019; Published: 7 April 2019

\begin{abstract}
This paper proposes a new designed trajectory tracking method for a hydraulic manipulator, which is the terminal sliding mode control with sliding perturbation observer (TSMCSPO). The dynamics of the hydraulic system are complex and uncertain, it also generates a large reaction force when working as an excavator or a dismantling robot. In this paper, the new control law is designed to force the trajectory of the hydraulic system to follow the reference despite complex dynamics, modeling error, the huge reaction force, and dynamic uncertainties. The sliding perturbation observer (SPO) in TSMCSPO estimates all disturbances from the outside environment, dynamic uncertainties, and modeling errors in real time. We included a simulation and an experiment to verify the approach, and to demonstrate the performance compared with other controllers (SMCSPO, SMC, and TSMC). Stabilities of SPO and TSMCSPO were analyzed based on the Lyapunov stability theory.
\end{abstract}

Keywords: sliding perturbation observer; hydraulic cylinder; terminal sliding mode control with sliding perturbation observer; robust controller; trajectory tracking

\section{Introduction}

Hydraulic actuators have more advantages than electric motors such as a large force output at high speed, high stiffness and durability, and rapid response [1,2]. Due to these advantages, hydraulic systems are widely used in many applications including construction, mining, and nuclear industries, where semi-automatic control systems are being adopted as means of improving the efficiency, quality, and safety of operations. Taylor et al. [3] designed a manipulator using hydraulic actuators for nuclear decommissioning.

However, hydraulic systems are more complex than electrical systems due to their highly nonlinear dynamics caused by phenomena such as fluid compressibility, nonlinear servo valve flow pressure characteristics, and dead band due to the internal leakage and hysteresis [4]. Besides its nonlinear dynamics, the main challenge of developing a control system for a hydraulic manipulator is the reaction force generated from the environment when it works as an excavator or dismantling manipulator. To obtain outstanding trajectory tracking performance, much of the research uses nonlinear control methods to compensate for the nonlinear features of an electro-hydraulic system. Furthermore, torque/force sensors are always used to measure the payload or reaction force from the environment. Sliding mode control is the most widely used nonlinear control algorithm for a hydraulic system with bounded disturbances, nonlinear terms, and system uncertainties. For example, in Reference [5], 
a sliding mode control with varying boundary layers is used for an electro-hydraulic position servo system. Rath et al. [6] used a non-singular higher order terminal sliding mode approach for vehicle suspension control with a hydraulic actuator. Adaptive control is also widely used for hydraulic actuators in combination with sliding mode control for varying dynamics and disturbances. Many researchers apply adaptive control to overcome system parameter variations, and sliding mode control is used as an effective way for controlling nonlinear systems with uncertainties. However, precise and detailed modeling of the system is needed to adopt the update law to compensate for the variable dynamic parameters. For instance, a sliding mode control with adaptive update law for a hydraulic system with unknown nonlinear parameters is proposed in [7]. In [8], a sliding mode control with adaptive update law of uncertain load disturbances is used to control a hydraulic parallel robot. The adaptive update law is used to update the system's dynamic parameters with a detailed and precise dynamic model which contains the bulk modulus of the fluid, the coefficient of internal leakage, etc. In [9], an adaptive control scheme based friction disturbance compensator structure was proposed for motion control, which approximates the friction term with RBF-type neural network. However, in adaptive control, the dynamic modeling should be built complicatedly or precisely for adapting the adaptation law, which is difficult to design and implement in a real system [10]. In recent years, much research also applies the adaptive algorithm on the sliding mode control named as adaptive SMC (sliding mode control). Some adaptive-gain-based sliding mode control are mentioned for more robustness, which selects the adaptive gain of the switching surface [11,12]. Some improved versions for an adaptive-robust controller with a time-delayed approach are shown in [13,14], which do not require any knowledge of system dynamic information. Initially, however, the no model based adaptive SMC showed a lot of tracking error in their results.

In this paper, we applied terminal mode control with a sliding perturbation observer (TSMCSPO) for a hydraulic system whose dynamics are only modeled as a simple second order system. The uncertainties, nonlinear terms, dynamic modeling errors of the system and disturbances are included by the definition of perturbation, which is estimated by the SPO (Sliding Perturbation Observer). Design for a real hydraulic system is more suitable than the adaptive updating law caused by simple dynamic modeling. The dynamic model of this hydraulic system is simplified as a 2nd order system. The identified system parameters are obtained by the signal compression method [15], which generates an equivalent impulse signal as the trajectory reference.

SPO is a combination of a sliding observer with a perturbation observer. Perturbation consists of disturbances, nonlinear terms, modeling errors, and system uncertainties. That is also effective at reducing the chattering in conventional SMC's caused by its robustness against the disturbance $[16,17]$, and improving the system's robustness by estimating and compensating for the perturbation $[18,19]$. SPO has been proven to be an accurate estimation within an interest designed for a low-frequency range through simulation $[20,21]$. Meanwhile, the estimated perturbation can be used to reduce chattering and tracking errors through SMC. SMCSPO (Sliding Mode Control with Sliding Perturbation Observer) is a combination of the controller SMC with SPO, which has an excellent performance in trajectory tracking. It only utilizes the partial state feedback to estimate the other states and perturbation including non-linear control terms. It has been proven to have a high tracking performance in some electrical motor applications (e.g., in [22,23] for a surgical robot). However, the convergence time of SMCSPO is slower than only an SMC with full states feed-back, and some undershoot occurs. This phenomenon is caused by the existing time delay of the sliding perturbation observer.

In order to reduce the disadvantage of time delay brought by SPO and have a robust tracking performance, TSMCSPO was designed and used in our current study. TSMCSPO combines TSMC (Terminal Sliding Mode Control) and SPO. TSMC uses the terminal sliding surface instead of the traditional linear sliding surface [24,25], resulting in the tracking error being lead to zero in a finite time fast. TSMC has been used and examined in control of robot and manipulators [26,27]. This fast controller is useful to speed up the convergence velocity around an equilibrium point. Due to the introduction of a non-linear sliding surface, preliminary TSMC may have a singularity problem, which 
makes it difficult to act on the actual mechanical application. In order to avoid the singularity problem, some methods have been developed and applied [28-30]. We use the method mentioned in [30] to avoid the singularity point in the TSMCSPO design.

In this research, the main point is the new TSMCSPO controller, which uses the terminal sliding mode to improve the slow convergence time and reduce the effect of time delay that occurs in the SPO. Application on the hydraulic system is novel using TMCSPO, though the SMC technical and perturbation observer is well known and not new for electric motors. The new designed controller is proposed for the first time in this paper. Then, the implementation using a hydraulic system is introduced with its dynamical analysis. Finally, the performance of this new controller TSMCSPO is demonstrated by MATLAB simulations compared with the conventional SMC, SMCSPO, and TSMC, as well as in real applications for the hydraulic servo system.

The remaining part of this paper is organized as follows. The controller design for a general robot manipulator system is described in Section 2. Section 3 introduces the hydraulic system and the application with TSMCSPO. A simulation carried out in MATLAB and the experimental result from the real hydraulic system are present in Sections 4 and 5, respectively. Section 6 concludes this work.

\section{Controller Design}

\subsection{System Description for a General Robot Manipulator}

The governing equation for a general second-order dynamic of the $j$-th link of a robot manipulator with n-degree-of-freedom (DOF) is described by the following differential equation:

$$
\begin{aligned}
& \ddot{x}_{j}=f_{j}(x)+\Delta f_{j}(x)+\sum_{i=1}^{n}\left[\left(b_{j i}(x)+\Delta b_{j i}(x)\right) u_{i}\right]+d_{j}(t) \\
& j=1, \ldots, n .
\end{aligned}
$$

where $x \triangleq\left[X_{1} \ldots X_{n}\right]^{T}$ is the state vector and $X_{j} \triangleq\left[x_{j}, \dot{x}_{j}\right]^{T}$. In practice, $x_{j}$ is the real trajectory for the $j$-th link. The terms $f_{j}(x)$ correspond to the nonlinear driving terms, while $\Delta f_{j}(x)$ corresponds to their uncertainties. The components $b_{j i}$ and $\Delta b_{j i}$ represent the elements of the control gain matrix and their uncertainties, while $d_{j}$ is the external disturbance and $u_{j}$ is the control input. The terms $f_{j}$ and $b_{j i}$ are assumed as well known continuous functions of state in literature [21]. Perturbation $\Psi_{j}(x, t)$ is defined as the combination of all the uncertainties in Equation (1): the physical meaning of perturbation includes the unidentified dynamic terms, the dynamic modeling error and the external disturbances of perturbation

$$
\Psi_{j}(x, t)=\Delta f_{j}(x)+\sum_{i=1}^{n}\left[\Delta b_{j i}(x) u_{i}\right]+d_{j}(t)
$$

In practice, the main component of the perturbation $\Psi_{j}(x, t)$ is the external disturbance from the environment for a general motor driven robot. However, for a hydraulic system, the dynamic uncertainties also have a big impact. The control goal is to drive state $x$ toward a desired state $x_{d} \triangleq\left[\boldsymbol{X}_{1 d} \ldots \boldsymbol{X}_{n d}\right]^{T}$ despite these perturbations.

Assumption 1. The perturbations have an upper bound by a known continuous function of the state:

$$
\Gamma_{j}(x, t)=F_{j}(x)+\sum_{i=1}^{n}\left|\Phi_{j i}(x) u_{i}\right|+D_{j}(t)>\left|\Psi_{j}(t)\right| .
$$

where $F_{j}>\left|\Delta f_{j}\right|, \Phi_{j i}>\left|\Delta b_{j i}\right|$ and $D_{j}>\left|d_{j}\right|$ represent the expected upper bounds of the uncertainties. 
The combination of a controller (TSMC) and an observer (SPO) results in a new form called terminal sliding mode control with sliding perturbation observer (TSMCSPO). The flow chart of TSMCSPO is shown in Figure 1.

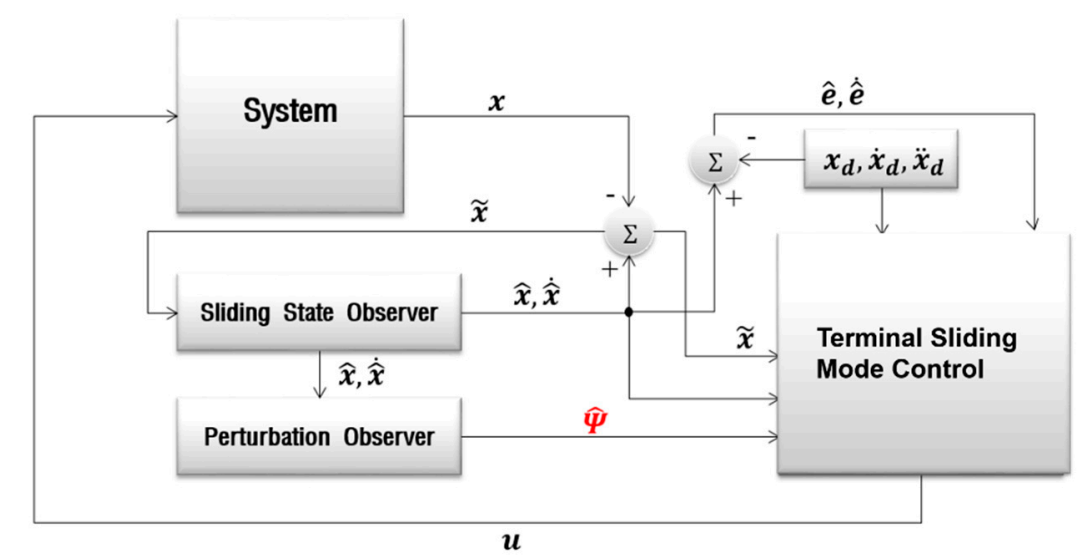

Figure 1. Flow chart of TSMCSPO (Terminal Sliding Mode Control with Sliding Perturbation Observer).

2.2. Sliding Perturbation Observer (SPO)

The state space with a new state variable $x_{3 j}$ can be represented as:

$$
\begin{gathered}
\dot{x}_{1 j}=x_{2 j} \\
\dot{x}_{2 j}=a_{3 j} \bar{u}_{j}+\Psi_{j} \\
\dot{x}_{3 j}=\alpha_{3 j} \dot{x}_{2 j}-\frac{\dot{\Psi}_{j}}{\alpha_{3 j}} \\
y_{j}=x_{1 j} .
\end{gathered}
$$

The new state variable $x_{3 j}$ is defined as:

$$
x_{3 j}=\alpha_{3 j} x_{2 j}-\frac{\Psi_{j}}{\alpha_{3 j}} .
$$

The sliding perturbation observer (SPO) equations for a $j$-th link can be described as follows:

$$
\begin{gathered}
\dot{\hat{x}}_{1 j}=\hat{x}_{2 j}-k_{1 j} \operatorname{sat}\left(\widetilde{x}_{1 j}\right), \\
\dot{\hat{x}}_{2 j}=\alpha_{3} \bar{u}_{j}-k_{2 j} \operatorname{sat}\left(\widetilde{x}_{1 j}\right)+\hat{\Psi}_{j} \\
\hat{\hat{x}}_{3 j}=\alpha_{3 j}^{2}\left(\bar{u}_{j}+\alpha_{3 j} \hat{x}_{2 j}-\hat{x}_{3 j}\right) \\
\hat{\psi}_{j}=\alpha_{3 j}\left(\alpha_{3 j} \hat{x}_{2 j}-\hat{x}_{3 j}\right) .
\end{gathered}
$$

In this equation, $\operatorname{sat}\left(\widetilde{x}_{1 j}\right)=\left\{\begin{array}{c}\widetilde{x}_{1 j}, i f\left|\widetilde{x}_{1 j}\right| \geq \varepsilon_{0 j} \\ \frac{\sqrt{x_{1 j}} \mid}{\widetilde{x}_{1 j}}, i f\left|\widetilde{x}_{1 j}\right| \leq \varepsilon_{0 j}\end{array} . \varepsilon_{0 j}\right.$ stands for the boundary layer of the SPO. The resulting error estimation dynamics becomes:

$$
\begin{gathered}
\dot{\tilde{x}}_{1 j}=\widetilde{x}_{2 j}-k_{1 j} \operatorname{sat}\left(\widetilde{x}_{1 j}\right) \\
\dot{\tilde{x}}_{2 j}=-k_{2 j} \operatorname{sat}\left(\widetilde{x}_{1 j}\right)+\widetilde{\Psi}_{j} \\
\dot{\bar{x}}_{3 j}=\alpha_{3 j}^{2}\left(\alpha_{3 j} \widetilde{x}_{2 j}-\widetilde{x}_{3 j}\right)+\frac{\dot{\Psi}}{\alpha_{3 j}} .
\end{gathered}
$$


The existence condition of the sliding mode to be satisfied is derived as:

$$
\begin{gathered}
\widetilde{x}_{2 j} \leq k_{1 j}\left(\text { if } \widetilde{x}_{1 j}>\varepsilon_{0 j}\right) \\
\widetilde{x}_{2 j} \geq-k_{1 j}\left(\text { if } \widetilde{x}_{1 j}<-\varepsilon_{0 j}\right) .
\end{gathered}
$$

where $k_{1 j}$ is a constant and larger than the maximum of $\widetilde{x}_{2 j}$, i.e., $k_{1 j}>\left|\max \left(\widetilde{x} 2_{j}\right)\right|$. After the observer sliding mode begins $\left(\widetilde{x}_{1 j}=0\right), \widetilde{x}_{2 j}$ dynamics becomes:

$$
\dot{\tilde{x}}_{2 j}+\left(\frac{k_{2 j}}{k_{1 j}}\right) \widetilde{x}_{2 j}=\widetilde{\Psi}_{j}
$$

It is desirable to place the breakpoint $\frac{k_{2 j}}{k_{1 j}}$ as high as possible in order to increase the attenuation from $\widetilde{\Psi}_{j}$ to $\widetilde{x}_{2 j}$, and improve the estimation accuracy of $x_{2 j}$ at the same time. If we select that $k_{2 j}$ is larger than the maximum of $\widetilde{\Psi}_{j}$, i.e., $k_{2 j} \geq\left|\max \left(\widetilde{\Psi}_{j}\right)\right|$, it can assure the steady state $\left|\widetilde{x}_{2_{j}}\right| \leq k_{1 j}$ from Equation (9), will force the states to stay in the sliding region. When $\left|\widetilde{x}_{1 j}\right| \leq \varepsilon_{0 j}$, the error estimation dynamics becomes:

$$
\begin{gathered}
\dot{\tilde{x}}_{1 j}=\widetilde{x}_{2 j}-k_{1 j} \frac{\widetilde{x}_{1 j}}{\varepsilon_{0 j}} \\
\dot{\tilde{x}}_{2 j}=-k_{2 j}{\widetilde{x_{1 j}}}_{\varepsilon_{0 j}}+\widetilde{\Psi}_{j} .
\end{gathered}
$$

Remark 1. If the gain $\alpha_{3 j}$ is selected high enough, the term $\frac{\dot{\Psi}_{j}}{\alpha_{3 j}}$ gives very little influence on the changes of $\widetilde{x}_{3 j}$ in Equation (7). $\tilde{x}_{3 j}$ also converges to 0 after $\tilde{x}_{2 j}$ reaches its equilibrium point (i.e., $\tilde{x}_{2 j}=0$ ) when $\frac{\dot{\Psi}_{j}}{\alpha_{3 j}}$ is small and can be neglected with a large selected value of $\alpha_{3 j}$. This can be proven using Lyapunov stability theory when both sides of Equation (7) are multiplied with $\widetilde{x}_{3 j}$.

Using Equations (4) and (5), the relation between $\widetilde{\Psi}_{j}$ and $\Psi_{j}$ in the Laplace domain is (see the Appendix A):

$$
\widetilde{\Psi}_{j}(p)=\frac{p\left[p^{2}+\left(k_{1 j} / \varepsilon_{0 j}\right) p+k_{2 j} / \varepsilon_{0 j}\right]}{p^{3}+\left(k_{1 j} / \varepsilon_{0 j}\right) p^{2}+\left(k_{2 j} / \varepsilon_{0 j}\right) p+\alpha_{3 j}^{2}\left(k_{2 j} / \varepsilon_{0 j}\right)}\left(-\Psi_{j}(p)\right) .
$$

This relation in Equation (11), between the real perturbation and the error of perturbation estimation, is a high pass filter. It means if the perturbation is in the low-frequency area the observer will estimate accurately. Using Equations (10) and (11), a relation between $\widetilde{x}_{2 j}$ and $\Psi_{j}$ in the Laplace domain is derived as:

$$
\widetilde{x}_{2 j}=\frac{p\left(p+\frac{k_{1 j}}{\varepsilon_{0 j}}\right)}{p^{3}+\left(\frac{k_{1 j}}{\varepsilon_{0 j}}\right) p^{2}+\left(\frac{k_{2 j}}{\varepsilon_{0 j}}\right) p+\alpha_{3 j}^{2}\left(\frac{k_{2 j}}{\varepsilon_{0 j}}\right)}\left(-\Psi_{j}(p)\right) .
$$

Only utilizing the partial state feedback, the estimated perturbation by the SPO can be used to reduce the effect of real perturbation for a more accurate estimation [21]. Moreover, the more accurate estimation can be used in the sliding mode control to follow the desired state.

When $\left|\widetilde{x}_{1 j}\right|<\varepsilon_{0 j}$, the saturation function sat $\left(\widetilde{x}_{1 j}\right)$ is reduced to $\frac{\widetilde{x}_{1 j}}{\varepsilon_{0 j}}$ as shown in Equation (10). The dynamics of the error state estimation shown in matrix form is:

$$
\left[\begin{array}{c}
\dot{\vec{x}}_{1 j} \\
\dot{\tilde{x}}_{2 j} \\
\dot{\vec{x}}_{3 j}
\end{array}\right]=\left[\begin{array}{ccc}
-\frac{k_{1 j}}{\varepsilon_{0 j}} & 1 & 0 \\
-\frac{k_{2 j}}{\varepsilon_{0 j}} & \alpha^{2}{ }_{3 j} & -\alpha_{3 j} \\
0 & \alpha^{3}{ }_{3 j} & -\alpha^{2}{ }_{3 j}
\end{array}\right] \times\left[\begin{array}{c}
\widetilde{x}_{1 j} \\
\widetilde{x}_{2 j} \\
\widetilde{x}_{3 j}
\end{array}\right]+\left[\begin{array}{l}
0 \\
0 \\
1
\end{array}\right] \frac{\dot{\psi}_{j}}{\alpha_{3 j}} .
$$


It shows that SPO is similar to a conventional Luenberger observer. The pole placement method is used to satisfy the stability and design the convergence time. The associated characteristic equation is:

$$
\left[\lambda^{3}+\left(\frac{k_{1 j}}{\varepsilon_{0 j}}\right) \lambda^{2}+\left(\frac{k_{2 j}}{\varepsilon_{0 j}}\right) \lambda+\alpha^{2}{ }_{3 j}\left(\frac{k_{2 j}}{\varepsilon_{0 j}}\right)\right]=0 .
$$

Let $p\left(\lambda_{d}\right)=\left(\lambda+\lambda_{d}\right)^{3}$ be the desired characteristic polynomial; this brings out the following design solutions:

$$
\begin{gathered}
\frac{k_{1 j}}{\varepsilon_{0 j}=3 \lambda_{d}} \\
\frac{k_{2 j}}{k_{1 j}=\lambda_{d}} \\
\alpha_{3 j}=\sqrt{\frac{\lambda_{d}}{3}} .
\end{gathered}
$$

The transfer function of Equation (11) turns out to be as follows:

$$
\begin{gathered}
\widetilde{\Psi}_{j}(p)=\frac{p\left[p^{2}+3 \lambda_{d} p+3 \lambda_{d}^{2}\right]}{\left(p+\lambda_{d}\right)^{3}}\left(-\Psi_{j}(p)\right) \\
\frac{\hat{\Psi}_{j}}{\Psi_{j}}=1+\frac{\widetilde{\Psi}_{j}}{\Psi_{j}}=\frac{\lambda^{3}{ }_{d}}{\left(p+\lambda_{d}\right)^{3}} .
\end{gathered}
$$

Remark 2. It shows the $\lambda_{d}$ is a design parameter, which determines the bandwidth of the high filter between the perturbation estimation error and real perturbation while a low pass filter relation is between estimated perturbation and real perturbation. The precision of estimated perturbation is restrained to only the slow motion mode.

An example of the effect of $\lambda_{d}$ on Equation (16) is shown by the bode diagram in Figure 2 where we give a different value to $\lambda_{d}$. The red dash line presents $\lambda_{d}=10$, and the solid blue line is $\lambda_{d}=20$. The results of Figure 2 show that when $\lambda_{d}$ increased, the estimated perturbation was much closer to the real perturbation. Although in terms of reducing errors and observing accuracy, a large gain $\lambda_{d}$ is required. The breaking point of the sliding observer dynamics inside a manifold cannot exceed $\frac{1}{5 \tau^{h w}}$ as shown by Moura et al. [21]. Where $\tau^{h w}$ is considered the dominant time delay depending on sampling time in the hardware. A similar proposition is also presented by Slotine and $\mathrm{Li}$ [16]. When reaching the sliding phase, the dynamics of the observer becomes $\dot{\bar{x}}_{1 j}=\widetilde{x}_{2 j}-\frac{k_{1 j}}{\varepsilon_{0 j}} \widetilde{x}_{1 j}$. The breaking point from the dynamics of the observer becomes $\frac{k_{1 j}}{\varepsilon_{0 j}}$. Therefore, the best selection of $\lambda_{d}$ in Equation (16) is

$$
\lambda_{d}=\frac{1}{15 \tau^{h w}} .
$$

This simple entity defines the necessary SPO parameters based on the limitation of the hardware.

Assumption 2. $R_{\max }$ represents the maximum ratio between perturbation $\Psi_{j}(p)$ and $\widetilde{x}_{2 j}$ on some frequency as:

$$
R_{\max j}=\operatorname{MAX}\left(\frac{p\left(p+3 \lambda_{d}\right)}{p^{3}+3 \lambda_{d} p^{2}+3 \lambda_{d}^{2} p+3 \lambda_{d}^{2} \alpha_{3 j}^{2}}\right) .
$$




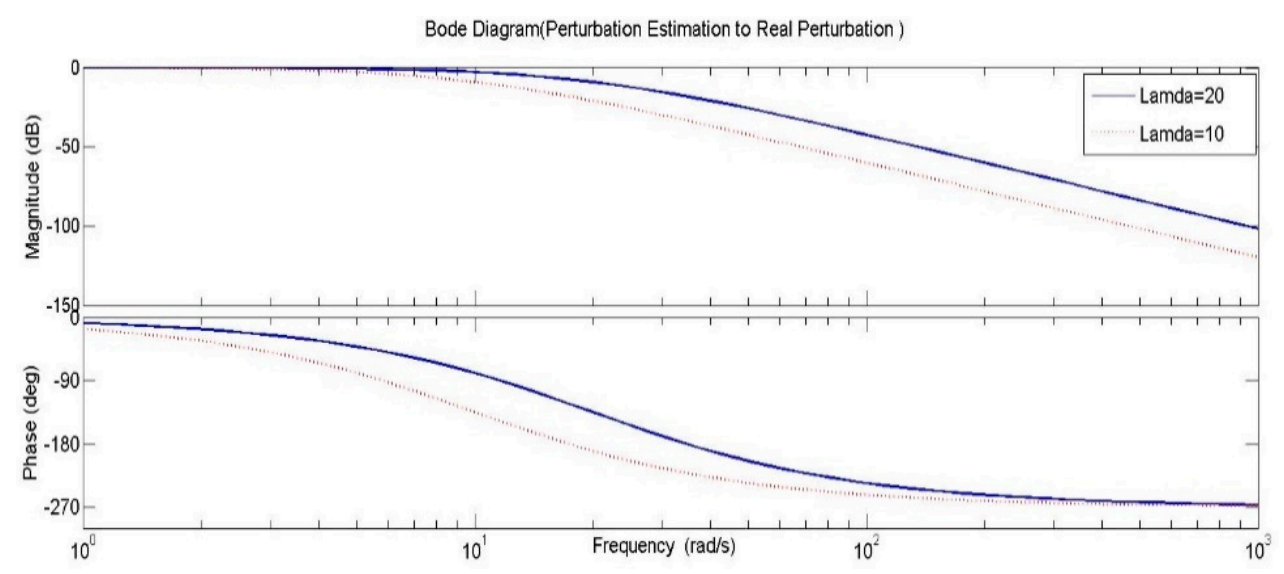

Figure 2. Bode diagram of estimated perturbation to real perturbation.

\subsection{Terminal Sliding Mode Control with Sliding Perturbation Observer (TSMCSPO)}

The new dynamics of $\hat{s}_{j}$ for TSMCSPO is defined as follows:

$$
\hat{s}_{j}=\dot{\hat{e}}_{j}+\beta_{j}\left|\hat{e}_{j}\right|^{r} \operatorname{sign}(\hat{e})
$$

where $\hat{e}_{j}=\hat{x}_{1 j}-x_{1 d j}$ is the estimated tracking error, and $\beta_{j}$ is positive constant. $\dot{\hat{s}}_{j}$ can be computed as:

$$
\dot{\hat{s}}_{j}=\alpha_{3 j} \bar{u}_{j}-\left[\frac{k_{2 j}}{\varepsilon_{0 j}}-\left(\frac{k_{1 j}}{\varepsilon_{0 j}}\right)^{2}\right] \widetilde{x}_{1 j}-\left(\frac{k_{1 j}}{\varepsilon_{0 j}}\right) \widetilde{x}_{2 j}-\ddot{x}_{1 j d}+\beta_{j} \frac{d}{d t}\left(\left|\hat{e}_{j}\right|^{r} \operatorname{sign}(\hat{e})\right)+\hat{\Psi}_{j} .
$$

The control input $\bar{u}_{j}$ of TSMCSPO is defined as:

$$
\bar{u}_{j}=\frac{1}{\alpha_{3 j}}\left\{\begin{array}{l}
-\Gamma_{j} R_{\max }\left(\frac{k_{1 j}}{\varepsilon_{0 j}}\right) \operatorname{sign}\left(\hat{s}_{j}\right)-\eta_{j}\left|\hat{s}_{j}\right|^{r} \operatorname{sign}\left(\hat{s}_{j}\right) \\
+\left[\frac{k_{2 j}}{\varepsilon_{0 j}}-\left(\frac{k_{1 j}}{\varepsilon_{0 j}}\right)^{2}\right] \widetilde{x}_{1 j}+\ddot{x}_{1 d j}-\hat{\Psi}_{j}-\beta_{j} \frac{d}{d t}\left(\left|\hat{e}_{j}\right|^{r} \operatorname{sign}(\hat{e})\right)
\end{array}\right\} .
$$

where $\dot{\hat{e}}=\hat{x}_{2 j}-\left(\frac{k_{1 j}}{\varepsilon_{0 j}}\right) \widetilde{x}_{1 j}-\dot{x}_{1 d j}$ is used in practical applications, $\eta_{j}$ is constant and positive, $R_{\max }$ and $\Gamma_{j}$ are defined in Assumptions 1 and 2. After reaching the sliding surface $\hat{s}_{j}=0$, the above function will be a non-singular function. However, if $s_{j} \neq 0, \dot{e}_{j} \neq 0$, and $e=0$, a singularity will occur [30]. To avoid the singularity problem, the following condition can be given as:

$$
\frac{d}{d t}\left(\left|\hat{e}_{j}\right|^{r} \operatorname{sign}(\hat{e})\right)=\left\{\begin{array}{c}
r|\hat{e}|^{r-1} \dot{\hat{e}}, \text { if } \dot{\hat{e}} \neq 0, \hat{e} \neq 0 \\
r|\Delta|^{r-1} \dot{\hat{e}}, \text { if } \dot{\hat{e}} \neq 0, \hat{e}=0 \\
0 \quad, \text { if } \dot{\hat{e}}=0, \hat{e}=0
\end{array}\right.
$$

where $\Delta>0$ is a small constant. Substituting $\bar{u}_{j}$ into (20), $\dot{\hat{s}}$ is computed as:

$$
\dot{\hat{s}}_{j}=-\Gamma_{j} R_{\max } j\left(\frac{k_{1 j}}{\varepsilon_{0 j}}\right) \operatorname{sign}\left(\hat{s}_{j}\right)-\eta_{3 j}\left|\hat{s}_{j}\right|^{r} \operatorname{sign}\left(\hat{s}_{j}\right)-\left(\frac{k_{1 j}}{\varepsilon_{0 j}}\right) \widetilde{x}_{2 j} .
$$

The Lyapunov description of TSMCSPO can be shown as:

$$
\dot{V}=\dot{\hat{s}} \hat{s}=\hat{s}\left(-\Gamma_{j} R_{\max } j\left(\frac{k_{1 j}}{\varepsilon_{0 j}}\right) \operatorname{sign}\left(\hat{s}_{j}\right)-\eta_{3 j}\left|\hat{s}_{j}\right|^{r} \operatorname{sign}\left(\hat{s}_{j}\right)-\left(\frac{k_{1 j}}{\varepsilon_{0 j}}\right) \widetilde{x}_{2 j}\right) \leq-\eta_{3 j} 2^{(r+1) / 2} V^{(r+1) / 2} .
$$




\section{TSMCSPO for the Hydraulic System}

\subsection{Hydraulic Servo System and Dynamics}

The hydraulic manipulator is shown in Figure 3 with its 3 dimensional file. It consists of three arms, two single-rod hydraulic actuated cylinders, and one AC servo motor. In this paper, only a 1st link hydraulic system is applied to evaluate the control performance by the simulation and experiment. The constitution of the 1st link is shown in Figure 4. It consists of an asymmetric piston, servo valve, an aluminum link, and an encoder. The rotation of link 1 is derived from the movement of the piston.

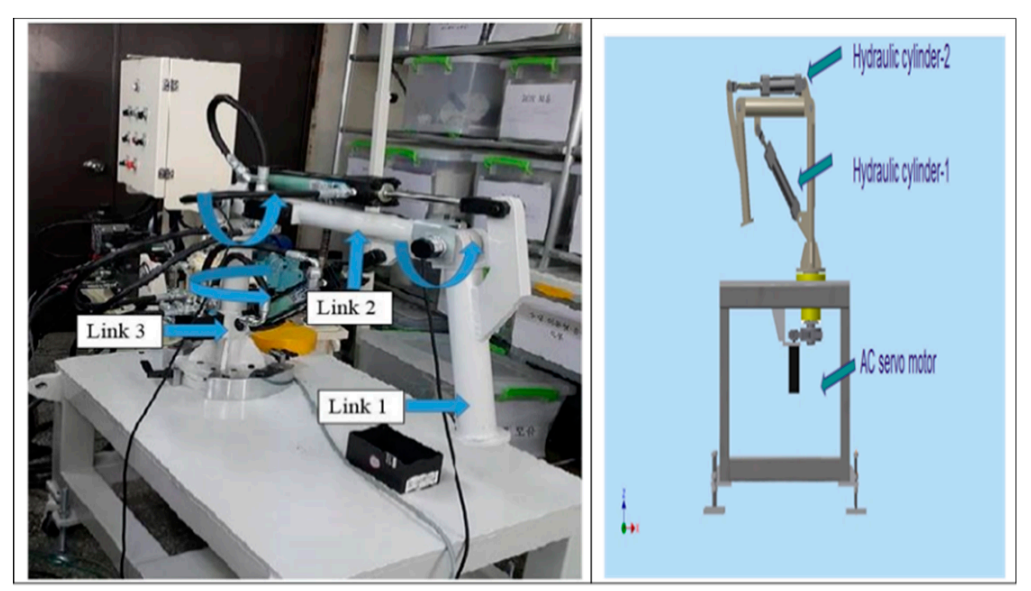

Figure 3. Hydraulic servo system with its 3 dimensional model.

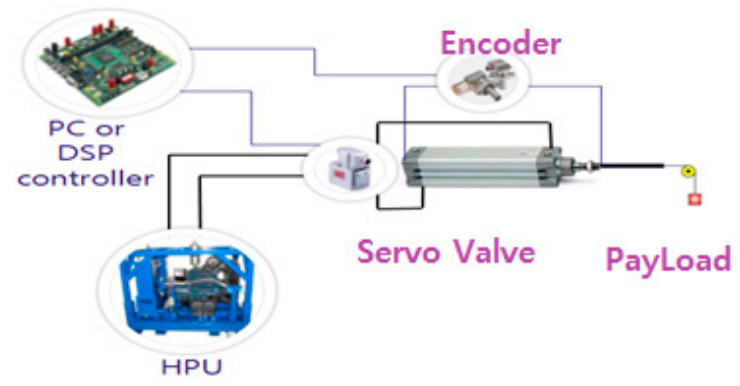

Figure 4. Schematic diagram of hydraulic system.

The rotation of link 1 was derived by the movement of the piston. The relation between the displacement of the piston and rotation angle of link1 are obtained as follows:

$$
\Delta x_{L} \approx R \Delta \theta_{1}
$$

where $x_{L}$ is the displacement of the piston, $\theta_{1}$ is the rotation angle of link 1 , and $R$ is the radius of link 1 's rotation (Figure 5).

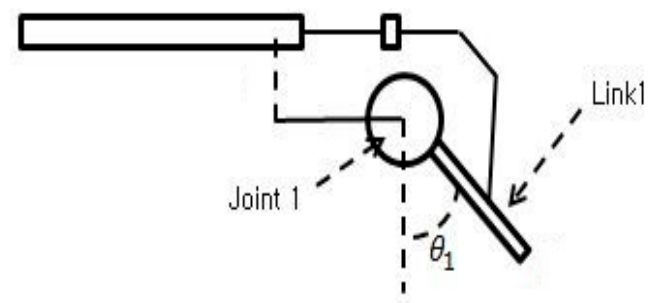

Figure 5. Schematic diagram of link 1. 
The dynamics of a single-rod hydraulic servo system was obtained as a state-space form in the following $[8,31]$ :

$$
\begin{gathered}
x=\left[x_{1}, x_{2}\right]^{T} \triangleq\left[x_{L}, \dot{x}_{L}\right]^{T} \\
\dot{x}_{1}=x_{2} \\
\dot{x}_{2}=\frac{1}{m}\left(A_{1} P_{1}-A_{2} P_{2}-b x_{2}-F_{d}\right) \\
\dot{P}_{1}=\frac{\beta_{e}}{V_{1}}\left(\left[-A_{1} x_{2}+k_{a} k_{q} \sqrt{\Delta P_{1}} u-C_{t}\left(P_{1}-P_{2}\right)\right]\right. \\
\dot{P}_{2}=\frac{\beta_{e}}{V_{2}}\left(\left[-A_{2} x_{2}-k_{a} k_{q} \sqrt{\Delta P_{2}} u-C_{t}\left(P_{1}-P_{2}\right)\right]\right.
\end{gathered}
$$

where $m$ is the mass of the piston, $P_{1}$ and $P_{2}$ are the pressures inside the two chambers of the cylinder, $b$ is the coefficient of viscous friction, $F_{d}$ contains the payload and the gravitational effects, $\beta_{e}$ is the bulk modulus of the fluid, $C_{t}$ is the internal leakage coefficient, $V_{1}$ and $V_{2}$ are the total fluid volumes of the two sides of the cylinder, $A_{1}$ and $A_{2}$ are the areas of the two sides of the piston, $k_{q}$ is the flow gain coefficient of the servo valve, $u$ is the servo valve control input signal, and $k_{a}$ is the servo amplifier gain [8].

When the leakage is negligible $P_{1}$ and $P_{2}$ can be obtained as:

$$
\begin{aligned}
& P_{1}\left(t_{1}\right)=u \int_{t_{0}}^{t_{1}} \frac{\beta_{e}}{V_{1}} k_{a} k_{q} \sqrt{\Delta P_{1}} d t-\int_{t_{0}}^{t_{1}} \frac{\beta_{e}}{V_{1}} A_{1} x_{2} d t+P_{1}\left(t_{0}\right) \\
& P_{2}\left(t_{1}\right)=u \int_{t_{0}}^{t_{1}} \frac{\beta_{e}}{V_{2}} k_{a} k_{q} \sqrt{\Delta P_{2}} d t-\int_{t_{0}}^{t_{1}} \frac{\beta_{e}}{V_{2}} A_{2} x_{2} d t+P_{2}\left(t_{0}\right)
\end{aligned}
$$

where $t_{0}$ is starting time, $P_{1}\left(t_{0}\right)$ and $P_{2}\left(t_{0}\right)$ are the initial pressures of each cylinder.

Substituting Equation (29) into Equation (27), we get:

$$
\begin{aligned}
\dot{x}_{2}= & \frac{1}{m}\left(A_{1} P_{1}-A_{2} P_{2}-b x_{2}-F_{d}\right)=u\left[\frac{A_{1}}{m} \frac{\beta_{e}}{V_{1}} k_{a} k_{q} \int_{t_{0}}^{t_{1}} \sqrt{\Delta P_{1}} d t-\frac{A_{2}}{m} \int_{t_{0}}^{t_{1}} \frac{\beta_{e}}{V_{2}} k_{a} k_{q} \sqrt{\Delta P_{2}} d t\right] \\
& -\frac{\beta_{e}}{V_{1}} \frac{A_{1}}{m} A_{1} \int_{t_{0}}^{t_{1}} x_{2} d t+\frac{A_{2}}{m} \frac{\beta_{e}}{V_{2}} A_{2} \int_{t_{0}}^{t_{1}} x_{2} d t-\frac{b}{m} x_{2}+\frac{A_{1}}{m} P_{1}\left(t_{0}\right)-\frac{A_{2}}{m} P_{2}\left(t_{0}\right)-\frac{F_{d}}{m}
\end{aligned}
$$

Assumption 3. The two sides cylinders have the same volume $V_{\text {eq }}$ and the same area $A_{\text {eq. }}$. Equation (30) can be simplified as follows:

$$
\dot{x}_{2}=u \frac{A_{e q}}{m} \frac{\beta_{e}}{V_{e q}} k_{a} k_{q}\left(P^{*}\right)-\frac{b}{m} x_{2}+\frac{A_{e q}}{m}\left[P_{1}\left(t_{0}\right)-P_{2}\left(t_{0}\right)\right]-\frac{F_{d}}{m}
$$

where $P^{*}$ is a constant which is assumed as an average value of $\left(\int_{t_{0}}^{t_{1}} \sqrt{\Delta P_{1}} d t-\int_{t_{0}}^{t_{1}} \sqrt{\Delta P_{2}} d t\right)$. Hence, the dynamics of the entire system can be considered as a SISO (Single-Input and Single-Output) second order system with the control input $u$ and the rotation output $\theta_{1}$ of link 1 as follows:

$$
\ddot{\theta}_{1}=\frac{1}{J_{1}} u-\frac{1}{J_{1}} D_{1} \dot{\theta}_{1}-\Psi .
$$

where $\Psi, D_{1}$ and $J_{1}$ is defined as follows:

$$
\begin{gathered}
\frac{1}{J_{1}}=\frac{A_{\text {eq }}}{m} \frac{\beta_{e}}{V_{\text {eq }}} k_{a} k_{q} P^{*} \\
\frac{D_{1}}{J_{1}}=\frac{b}{m} \\
\Psi=-\frac{A_{\text {eq }}}{m}\left[P_{1}\left(t_{0}\right)-P_{2}\left(t_{0}\right)\right]+\frac{F_{d}}{m}+\text { Model Assumption Errors }
\end{gathered}
$$

For a second order system in Equation (32), $J_{1}$ is considered as the inertia of the system, $D_{1}$ contains the damping term, and $\Psi$ is defined as the perturbation which contains modeling errors, nonlinear terms, some simplified calculation error, and main disturbances from the environment. The perturbation $\Psi$ is estimated using SPO. The details of SPO are shown in the next section. 
Remark 3. The whole system in Equation (32) contains a hydraulic pump, a control valve, and a hydraulic cylinder. Control input is given by an MMC PCI board which outputs a voltage to the servo valve, and system output is the rotation angle of the link forced by the placement of a hydraulic actuator piston. The sampling time is set as $2 \mathrm{~ms}$, which is fast enough to apply the control algorithm directly without analyzing by digital control theory.

\subsection{TSMCSPO for Hydraulic System}

Applying the proposed controller in Equation (21), the control input of TSMCSPO for the end-effector of a hydraulic cylinder is shown as:

$$
\begin{gathered}
u_{t s m c s p o}=J_{1} \alpha_{3} \bar{u}+D_{1} \hat{\theta}_{12} \\
\bar{u}_{t s m c s p o}=\frac{1}{\alpha_{3}}\left\{-\Gamma_{1} R_{\max 1}\left(\frac{k_{1}}{\varepsilon_{01}}\right) \operatorname{sign}\left(\hat{s}_{1}\right)-\eta_{1}\left|\hat{s}_{1}\right|^{r} \operatorname{sign}\left(\hat{s}_{1}\right)+\left[\frac{k_{2}}{\varepsilon_{01}}-\left(\frac{k_{1}}{\varepsilon_{01}}\right)^{2}\right] \widetilde{\theta}_{1}+\ddot{\theta}_{1 d}\right. \\
\left.-\beta_{1} r|\hat{e}|^{r-1}\left(\hat{\theta}_{12}-\left(\frac{k_{1}}{\varepsilon_{0}}\right) \widetilde{\theta}_{1}-\dot{\theta}_{1 d}\right)-\hat{\Psi}\right\} .
\end{gathered}
$$

when $|\hat{e}|=0$,

$$
\begin{gathered}
\bar{u}_{t s m c s p o}=\frac{1}{\alpha_{3}}\left\{-\Gamma_{1} R_{\max 1}\left(\frac{k_{1}}{\varepsilon_{01}}\right) \operatorname{sign}\left(\hat{s}_{1}\right)-\eta_{1}\left|\hat{s}_{1}\right|^{r} \operatorname{sign}\left(\hat{s}_{1}\right)+\right. \\
\left.\left[\frac{k_{2}}{\varepsilon_{01}}-\left(\frac{k_{1}}{\varepsilon_{01}}\right)^{2}\right] \widetilde{\theta}_{1}+\ddot{\theta}_{1 d}-\beta_{1} r|\Delta|^{r-1}\left(\hat{\theta}_{12}-\left(\frac{k_{1}}{\varepsilon_{o}}\right) \widetilde{\theta}_{1}-\dot{\theta}_{1 d}\right)-\hat{\Psi}\right\} .
\end{gathered}
$$

where $\theta_{12}$ is the rotation velocity of link $1, \hat{e}=\hat{\theta}_{1}-\theta_{1 d}, \widetilde{\theta}_{1}=\hat{\theta}_{1}-\theta_{1} \cdot \dot{\theta}_{1 d}$ and $\ddot{\theta}_{1 d}$ are the desired velocity and acceleration of link 1 , respectively. After reaching the sliding surface, $\dot{\hat{e}}=\hat{\theta}_{12}-\left(\frac{k_{1}}{\varepsilon_{0}}\right) \widetilde{\theta}_{1}-\dot{\theta}_{1 d}$. The term $|e|^{r-1}$ in TSMCSPO creates a singularity problem and can be solved by the condition in Equation (22).

\section{Simulations}

This section introduces a trajectory tracking simulation in MATLAB to verify the performance of TSMCSPO.

The desired trajectory used in this simulation is shown in Figure 6. The desired trajectory includes all possible response inputs (i.e., ramp, sinusoidal, and step). It increased until it reached 45 degrees at $19.7 \mathrm{~s}$. At the start, the trajectory gradually increased by the ramp input until $5 \mathrm{~s}$. Then, it increased by a mix of sinusoidal input $(3 \mathrm{rad} / \mathrm{s}, 0.477 \mathrm{~Hz})$ and ramp input until it reached its maximum value (i.e., 45 degrees). It then dropped until it reached 15 degrees at $25 \mathrm{~s}$. From $25 \mathrm{~s}$, the reference input was designed as a step input. During all the movement, the external force is set as:

$$
\Psi=\mathrm{h} \cdot x_{1} .
$$

where $x_{1}$ is the real trajectory, $\mathrm{h}$ is a positive number, and the maximum frequency of perturbation is considered as $3 \mathrm{rad} / \mathrm{s}$, which is assumed as the real operation frequency. Table 1 reports the parameters used in the simulations. $\alpha_{3}, \frac{k_{1}}{\varepsilon_{01}}$ and $k_{2 j}$ can be determined by $\lambda_{d}$ shown in Equation (15).

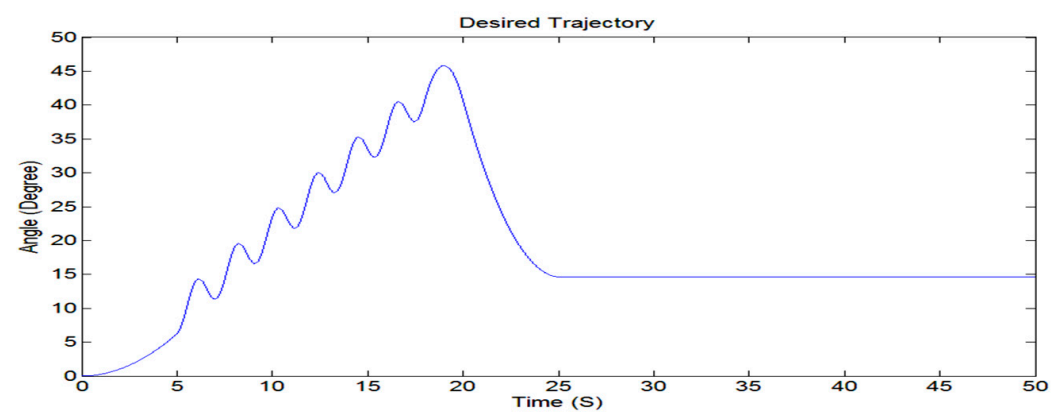

Figure 6. Desired trajectory. 
Table 1. Parameter selections of simulation and experiment.

\begin{tabular}{cl}
\hline Parameters & Values \\
\hline$\beta_{1}, \lambda_{d}, \eta_{1}$ & 10 \\
\hline$\varepsilon_{c}, \mathrm{~h}$ & 1 \\
\hline$r$ & 0.7 \\
\hline$\Delta$ & 0.01 \\
\hline
\end{tabular}

The fractional power term $\dot{\hat{e}}|\hat{e}|^{r-1}$ in the control law of TSMCSPO can be seen as the term that bridges between linear control $(r \rightarrow 1)$ and nonlinear control $(r \rightarrow 0)$. If we increase the ratio $(r \rightarrow 0)$, the convergence time is reduced. Otherwise, the chattering will be much more severe. Moreover, in the simulation, the parameter $\eta_{1}$ is selected to be larger than the boundary of the expected perturbation, which is proposed in the previous section. Because the maximum frequency of the disturbance is given as $3 \mathrm{rad} / \mathrm{s}$ (oscillation part in the desired trajectory) $\lambda_{d}$ can be selected as 10 when the bandwidth is near $5 \mathrm{rad} / \mathrm{s}$ in the sliding perturbation observer.

Figure 7 shows the simulation result of the error trajectory comparison between the proposed control scheme and other three controllers. The other three controllers were: (a) SMC designed as $u_{s m c}=-J_{1} K_{1} s a t(s)+D_{1} \theta_{12}+J_{1} \ddot{\theta}_{1 d}-J_{1} c \dot{e}$ with parameters $K_{1}=10$ and $c=10$; (b) SMCSPO used in Equation A6 from Reference [32] with same parameters in SPO and $K_{1}=10$; and (c) conventional TSMC described in [28] $u_{t s m c}=-\eta_{2} J_{1} \operatorname{sign}(s)-\eta_{3} J_{1}|s|^{r} \operatorname{sign}(s)+D_{1} \theta_{12}+J \ddot{\theta}_{1 d}-J_{1} \beta r|e|^{r-1}(\dot{e})$ with parameters $\eta_{2}=0.6$ and $\eta_{3}=10$.

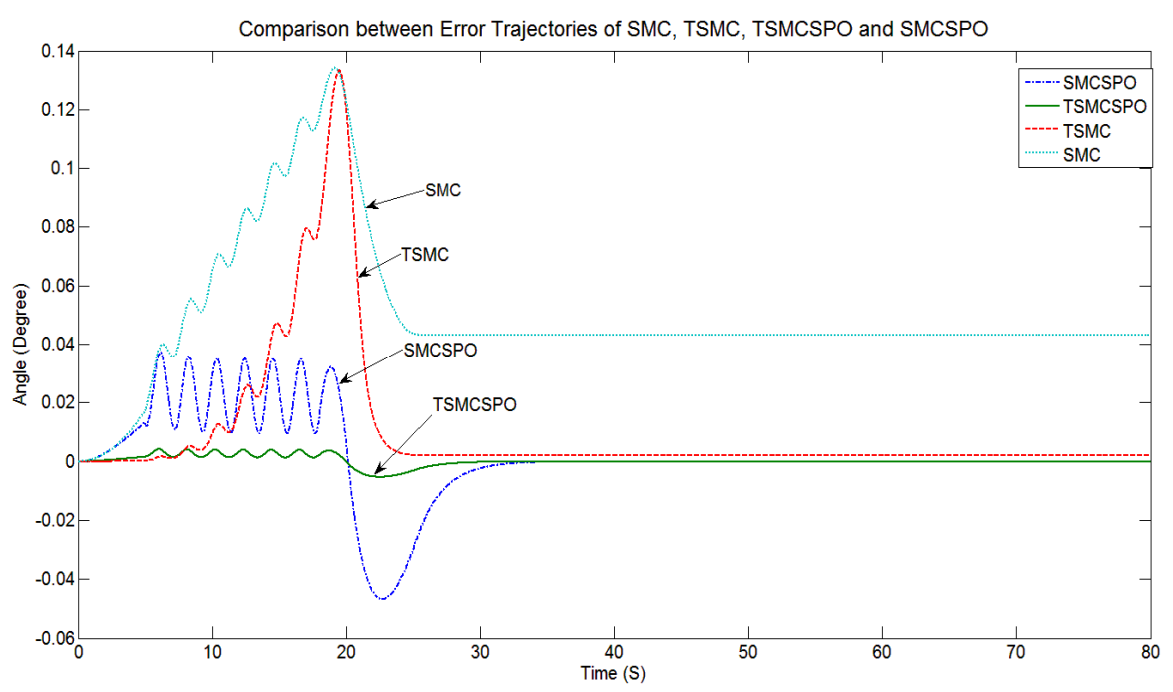

Figure 7. Trajectory Tracking Error Comparisons.

Figure 8 gives the control input result of SMCSPO and TSMCSPO. The blue line presents the control input of TSMCSPO, and the line shows that of SMCSPO. An enlarged part of the interval from 11.5 to $11.6 \mathrm{~s}$ result shows that the control input of TSMCSPO had more chattering than SMCSPO, but not too great. TSMC also had more chattering than SMC; their results almost overlapped-similar to the result of TSMCSPO and SMCSPO. The overlapping phenomenon was caused by the huge control input needed for moving the hydraulic system. The chattering resulted from the nonlinear terminal sliding surface. 


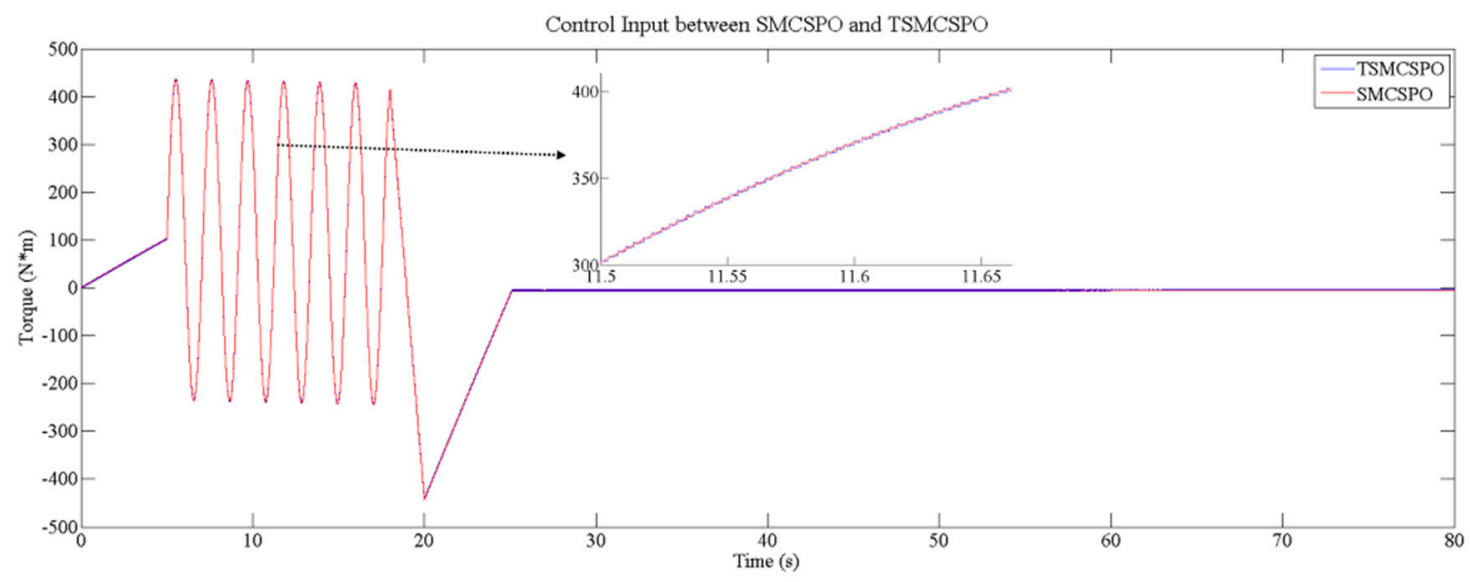

Figure 8. Comparison between error trajectories of SMC.

Comparing the error trajectories of SMC, TSMC, TSMCSPO, and SMCSPO is discussed in Table 2 which includes the maximum error, steady-state error, undershoot, and settling time. TSMC has a faster convergence time with less steady state error in the final step input section. This means TSMC has more attenuation than SMC. However, the results of both SMCSPO and TSMCSPO showed less error and 0 steady state error, which benefited from the SPO. In both SMCSPO and TSMCSPO, an undershoot error existed, which was caused by the time delay. This time delay can be explained by Equation (11). The different phases between $\hat{\Psi}_{j}$ and $\Psi_{j}$ (shown in Figure 2) resulted in time delay and undershoot errors. This also made the settling time of SMCSPO slower than that of SMC. Comparing the settling time and rising time, we found that the terminal mode made the convergence time faster, which is certified in both TSMC and TSMCSPO. Altogether, the TSMCSPO exhibited less error than others, a 0 steady state error that is better than that of TSMC and a faster response than SMCSPO.

Table 2. Performance parameters.

\begin{tabular}{ccccc}
\hline Controllers & SMC $^{\mathbf{a}}$ & SMCSPO $^{\mathbf{b}}$ & TSMC $^{\mathbf{c}}$ & TSMCSPO $^{\mathbf{d}}$ \\
\hline Maximum error (degree) & 0.134 & 0.0372 & 0.133 & 0.0042 \\
Steady state error (degree) & 0.0432 & 0 & 0.0021 & 0 \\
Undershoot max. error (degree) & - & 0.0466 & - & 0.0053 \\
Settling time (s) & 25.28 & 33.59 & 24.18 & 29.75 \\
\hline
\end{tabular}

a SMC: Sliding Mode Control; ${ }^{\text {b }}$ SMCSPO: Sliding Mode Control with Sliding Perturbation Observer; ${ }^{c}$ TSMC: Terminal Sliding Mode Control; ${ }^{\mathrm{d}}$ TSMCSPO: Terminal Sliding Mode Control with Sliding Perturbation Observer.

\section{Experiment}

The real hydraulic system was used to demonstrate the trajectory tracking performance of TSMCSPO. The experiment was implemented on link 1 of the hydraulic system using TSMCSPO and SMCSPO. We expected the hydraulic system had many nonlinearities and uncertainties, resulting in the controller without the perturbation observer having a bad performance. Assuming the boundary of perturbation in the real system is less than $\Gamma_{1}=1 V$ shown as the unit of voltage. It can be shown in the unit of torque as $\Gamma_{1} \cdot$ ratio $_{\text {servo-valve }}\left(\frac{N}{V}\right) \approx 2500 \mathrm{~N}$. Hence, the maximum of perturbation was assumed as $2500 \mathrm{~N}$. With the purpose of how the gain $\lambda_{d}$ affects the controller, the experiments were carried out twice with different $\lambda_{d}$ values. The design parameters of TSMCSPO and SMCSPO were the same as the previous simulation, only with different $\lambda_{d}$ values (i.e., 10 and 20). In the real experiment, chattering was reduced by using the saturation function $\operatorname{sat}\left(\hat{s}_{1}\right)$ instead of the term $\operatorname{sign}\left(\hat{s}_{1}\right)$ in Equation (34), which is defined as if $\left|\hat{s}_{j}\right| \geq \varepsilon_{c j} s a t\left(\hat{s}_{j}\right)=\frac{\hat{s}_{j}}{\left|\hat{s}_{j}\right|}$ and if $\left|\hat{s}_{j}\right| \leq \varepsilon_{c j} \frac{\hat{s}_{j}}{\varepsilon_{c j}}, \varepsilon_{c j}$ is positive constant, and $\varepsilon_{c 1}$ was selected as 0.2 to balance the steady state error and the chattering. Detailed analysis is shown in the Appendix A. 
The experimental result of the comparison between error trajectories of TSMCSPO and SMCSPO at the value of $\lambda_{d}$ was 10, as shown in Figure 9. The red line shows the tracking error result of TSMCSPO, and the blue dotted line shows the result of the tracking error using SMCSP. The maximum error of TSMCSPO was 0.292 degrees at $6 \mathrm{~s}$. The maximum error of SMCSPO was 0.985 degrees at $6.5 \mathrm{~s}$. In the step response area, the small chattering was caused by the revolution of the encoder.

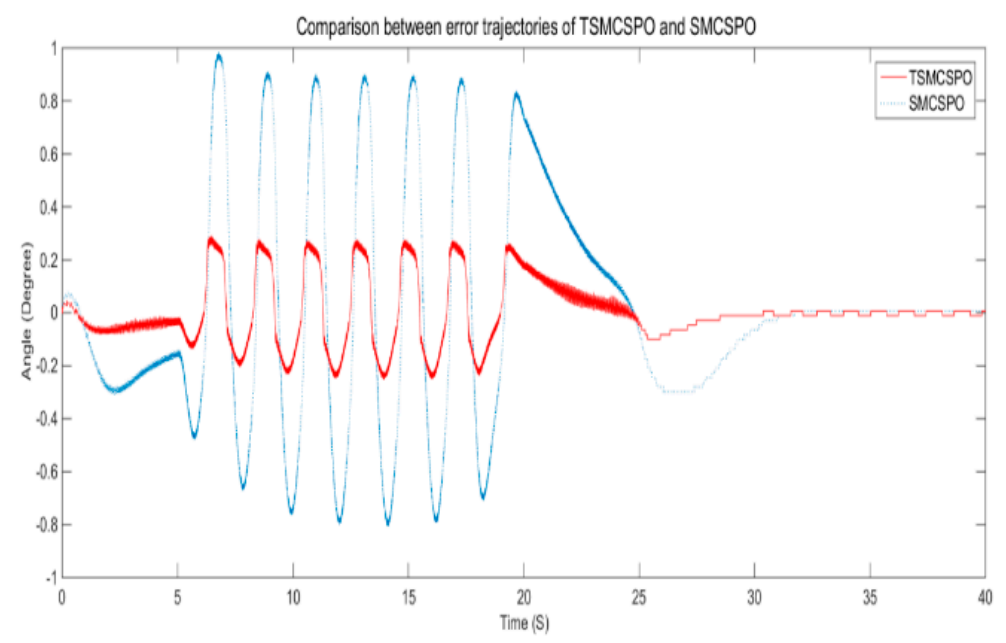

Figure 9. Comparing error trajectories of TSMCSPO and SMCSPO $\left(\lambda_{d}=10\right)$.

Figure 10 shows the experimental result of comparing the error trajectories of TSMCSPO and SMCSPO, where the value of $\lambda_{d}$ was 20. The red line shows the experimental result of TSMCSPO error, and the blue dotted line shows the experimental result for the error of SMCSPO. The maximum error of TSMCSPO was 0.2285 degrees at $17.15 \mathrm{~s}$, and the maximum error of SMCSPO was 0.6864 degrees at $17.4 \mathrm{~s}$.

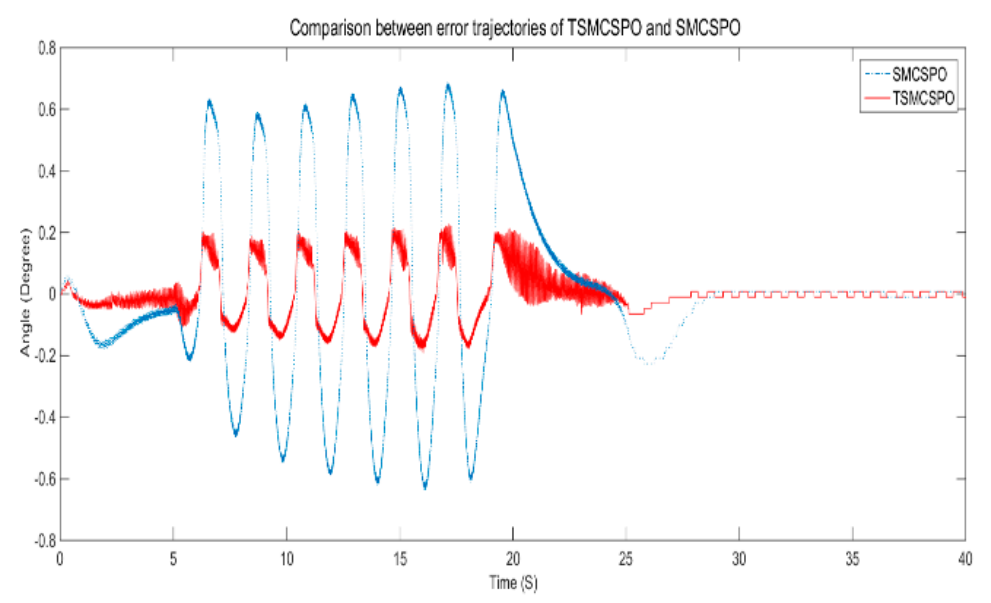

Figure 10. Comparison between error trajectories of TSMCSPO and SMCSPO $\left(\lambda_{d}=20\right)$.

Both SMCSPO and TSMCSPO had a 0 steady state error (the continuous oscillation is caused by the resolution of the encoder whose least measurable unit is 0.018 degree.). The trajectory tracking error of TSMCSPO was significantly less compared to that of SMCSPO in both experiments. The error of the trajectory also decreased when the value of $\lambda_{d}$ increased, with a small increase in chattering. The reason for this was that the phenomenon was affected by the increased switching of control input gain (i.e., $k_{1}$ in Equation (34)). These data indicate that TSMCSPO has a fast convergence time and less undershoot compared with SMCSPO. 


\section{Conclusions}

This research addresses the trajectory tracking control of a hydraulic actuated manipulator with a new designed controller, TSMCSPO. The modeling error, dynamics, nonlinear terms, and disturbances are defined as a variable state perturbation. SPO is used to estimate the perturbation. The main contribution of this research is the design of TSMCSPO and its implementation on a hydraulic system. In the simulation, TSMCSPO shows outstanding performance in trajectory tracking, with 0 state error compared to SMC and TSMC. TSMCSPO also has a faster convergence speed than SMCSPO, which improves on the convergence speed in spite of the bad effect from the time delay phenomenon that exists in SPO. The new approach to TSMCSPO also verified that it possesses faster convergence time and less undershoot compared to SMCSPO from the hydraulic system experiment.

Author Contributions: Conceptualization, J.W. and M.C.L.; Formal analysis, J.W., K.D.K.; Funding acquisition, M.C.L. and S.A.; Methodology, J.W.; Software J.W., K.D.K.; Supervision, M.C.L. and S.A.; Writing-original draft, J.W.; Writing-review \& editing, J.W., S.J.A., and K.D.K

Funding: This research was funded and conducted under the Competency Development Program for Industry Specialists of the Korean Ministry of Trade, Industry and Energy (MOTIE), Korea Institute for Advancement of Technology (KIAT) and the Ministry of Education (1345253125).

Acknowledgments: This research was supported under the Competency Development Program for Industry Specialists of the Korean Ministry of Trade, Industry and Energy (MOTIE), operated by Korea Institute for Advancement of Technology (KIAT). (No. P0008473, The development of high skilled and innovative manpower to lead the Innovation based on Robot), the Technology Innovation Program (10073147, Development of Robot Manipulation Technology by Using Artificial Intelligence) funded By the Ministry of Trade, Industry \& Energy (MOTIE, Korea) and the National Research Foundation of Korea (NRF) funded by the Ministry of Education (1345253125).

Conflicts of Interest: The authors declare no conflict of interest.

\section{Appendix A}

Proof of Equation (11):

First, using Equation (7), we can get:

$$
\widetilde{x}_{2 j}=\alpha_{3 j}^{-3}\left(\alpha_{3 j}{ }^{2} \widetilde{x}_{3 j}+\dot{\bar{x}}_{3 j}\right)-\frac{\dot{\Psi}}{\alpha^{4}{ }_{3 j}}
$$

Because the estimated perturbation is defined in Equation (6) as:

$$
\hat{\psi}_{j}=\alpha_{3 j}\left(\alpha_{3} \hat{x}_{2 j}-\hat{x}_{3 j}\right)
$$

$\widetilde{x}_{3 j}$ is simplified as:

$$
\begin{gathered}
\widetilde{x}_{3 j}=\hat{x}_{3 j}-\alpha_{3 j} x_{2 j}+\frac{\Psi_{j}}{\alpha_{3 j}} \\
=\hat{x}_{3 j}-\alpha_{3 j} x_{2 j}+\left(\frac{\hat{\Psi}_{j}-\widetilde{\Psi}_{j}}{\alpha_{3 j}}\right) \\
=-\alpha_{3 j} \widetilde{\psi}+\alpha_{3 j} \widetilde{x}_{2 j}
\end{gathered}
$$

Since SMCSPO is discussed in the manifold $\left|\hat{s}_{j}\right| \leq \varepsilon_{c j}$, from Equation (7) we can get:

$$
\begin{gathered}
\dot{\widetilde{x}}_{1 j}=\widetilde{x}_{2 j}-\frac{k_{1 j}}{\varepsilon_{0 j}} \widetilde{x}_{1 j} \\
\widetilde{x}_{1 j}=\frac{\left(-\dot{\bar{x}}_{2 j}+\widetilde{\Psi}_{j}\right)}{\frac{k_{2 j}}{\varepsilon_{0 j}}} \\
\dot{\tilde{x}}_{2 j}+\frac{k_{2 j}}{\varepsilon_{0 j}} \widetilde{x}_{1 j}=\widetilde{\Psi}_{j}
\end{gathered}
$$


The derivative of time in Equation (A6) is:

$$
\ddot{\widetilde{x}}_{2 j}+\frac{k_{2 j}}{\varepsilon_{0 j}} \dot{\vec{x}}_{1 j}=\dot{\widetilde{\Psi}}_{j}
$$

Substituting Equation (A5) in Equation (A7), we can get:

$$
\ddot{\widetilde{x}}_{2 j}+\frac{k_{2 j}}{\varepsilon_{0 j}}\left\{\widetilde{x}_{2 j}-\frac{k_{1 j}}{k_{2 j}}\left(-\dot{\widetilde{x}}_{2 j}+\widetilde{\Psi}_{j}\right)\right\}=\dot{\widetilde{\Psi}}_{j}
$$

Using Equations (A1) and (A2), Equation (A6) is rewritten as:

$$
-\ddot{\widetilde{\Psi}}_{j}-\overline{\widetilde{\Psi}}_{j}-\frac{k_{2 j}}{\varepsilon_{0 j}} \dot{\widetilde{\Psi}}_{j}-\frac{k_{2 j}}{\varepsilon_{0 j}} \dot{\Psi}_{j}-\frac{k_{1 j}}{\varepsilon_{0 j}} \ddot{\widetilde{\Psi}}_{j}-\frac{k_{1 j}}{\varepsilon_{0 j}} \ddot{\Psi}_{j}-\alpha_{3 j}^{4} \frac{k_{1 j}}{\varepsilon_{0 j}} \widetilde{\Psi}_{j}=\alpha_{3 j}^{4} \dot{\Psi}_{j}
$$

After s the Laplace transform, using Equation (A8) to substitute $\dot{\bar{x}}_{2 j}$ in Equation (15) for $\widetilde{\Psi}_{j}$, then Equation (16) can be derived.

Proof of Equation (24):

$$
\begin{gathered}
\dot{V}=\dot{\hat{s}}_{j} \hat{s}_{j}=\hat{s}_{j}\left(-\Gamma_{j} R_{\max }\left(\frac{k_{1 j}}{\varepsilon_{0 j}}\right) \operatorname{sign}\left(\hat{s}_{j}\right)-\eta_{3 j}\left|\hat{s}_{j}\right|^{r} \operatorname{sign}\left(\hat{s}_{j}\right)-\left(\frac{k_{1 j}}{\varepsilon_{0 j}}\right) \widetilde{x}_{2 j}\right) \\
=\hat{s}_{j}\left(-\Gamma_{j} R_{\max }\left(\frac{k_{1 j}}{\varepsilon_{0 j}}\right) \frac{\left|\hat{s}_{j}\right|}{\hat{s}_{j}}-\eta_{3 j}\left|\hat{s}_{j}\right|^{r} \frac{\hat{s}_{j} \mid}{\hat{s}_{j}}-\left(\frac{k_{1 j}}{\varepsilon_{0 j}}\right) \widetilde{x}_{2 j}\right) . \\
\left(\because \operatorname{sign}\left(\hat{s}_{j}\right)=\frac{\left|\hat{s}_{j}\right|}{\hat{s}_{j}} \operatorname{or} \frac{\hat{s}_{j}}{\hat{s}_{j} j}\right) \leq-\eta_{3 j}\left|\hat{s}_{j}\right|^{r+1} \\
\left(\because \Gamma_{j} R_{\max j}>\widetilde{x}_{2 j}\right) . \Rightarrow V \leq-\eta_{2 j} 2^{(r+1) / 2} V^{(r+1) / 2} .
\end{gathered}
$$

Stability analysis in the case of using a saturation function instead of sign function:

$$
\begin{gathered}
\dot{V}=\dot{\hat{s}}_{j} \hat{s}_{j}=\hat{s}_{j}\left(-\Gamma_{j} R_{\max } j\left(\frac{k_{1 j}}{\varepsilon_{0 j}}\right) \operatorname{sat}\left(\hat{s}_{j}\right)-\eta_{3 j}\left|\hat{s}_{j}\right|^{r} \operatorname{sat}\left(\hat{s}_{j}\right)-\left(\frac{k_{1 j}}{\varepsilon_{0 j}}\right) \widetilde{x}_{2 j}\right) \\
=\hat{s}_{j}\left(-\Gamma_{j} R_{\max }\left(\frac{k_{1 j}}{\varepsilon_{0 j}}\right) \frac{\hat{s}_{j}}{\varepsilon_{c j}}-\eta_{3 j}\left|\hat{s}_{j}\right|^{r} \frac{\hat{s}_{j}}{\varepsilon_{c j}}-\left(\frac{k_{1 j}}{\varepsilon_{0 j}}\right) \widetilde{x}_{2 j}\right) . \\
\left(\because\left|\hat{s}_{j}\right|<\varepsilon_{c j},\left|\frac{\hat{s}_{j}}{\varepsilon_{c j}}\right|<1\right)
\end{gathered}
$$

The requirement for the Lyapunov asymptotic stability is that $\frac{\Gamma_{j} R_{\max }\left|\hat{s}_{j}\right|}{\varepsilon_{c j}}>\widetilde{x}_{2 j}$. If we increase $\varepsilon_{c j}$, chattering is reduced due to the less scope of sign switching. However, the steady state error will have an increment caused by its instability when $\left|\hat{s}_{j}\right|$ is much less than $\varepsilon_{c j}$. In the real experiment, the steady state was discussed in an extremely low frequency. The value of $\frac{\Gamma_{j} R_{\max }}{\widetilde{x}_{2 j}}$ was much larger than 10, which was computed by using Equations (18) and (12). When we selected the value $\varepsilon_{c j}=0.2$, it guaranteed that $\left|\hat{j}_{j}\right|$ was less than 0.02 , which was assumed to be the hydraulic system's accuracy.

\section{References}

1. Dasgupta, B.; Mruthyunjaya, T.S. The Stewart platform manipulator: A review. Mech. Mach Theory 2005, 35, 15-40. [CrossRef]

2. Manring, N.; Roger, C. Hydraulic Control Systems; John Wiley \& Sons: New York, NY, USA, 2005.

3. Taylor, C.J.; Robertson, D. State-dependent control of a hydraulically actuated nuclear decommissioning robot. Control Eng. Pract. 2013, 21, 1716-1725. [CrossRef]

4. Zhu, W.H.; Piedboeuf, J.C. Adaptive output force tracking control of hydraulic cylinders with applications to robot manipulators. J. Dyn. Syst. Meas. Control 2005, 127, 206-217. [CrossRef]

5. Chen, H.M.; Renn, J.C.; Su, J.P. Sliding mode control with varying boundary layers for an electro-hydraulic position servo system. Int. J. Adv. Manuf. Technol. 2005, 26, 117-123. [CrossRef] 
6. Rath, J.J.; Defoort, M.; Karimi, H.R.; Veluvolu, K.C. Output feedback active suspension control with higher order terminal sliding mode. IEEE Trans. Ind. Electron. 2017, 64, 1392-1403. [CrossRef]

7. Guan, C.; Pan, S. Adaptive sliding mode control of electro-hydraulic system with nonlinear unknown parameters. Control Eng. Pract. 2008, 16, 1275-1284. [CrossRef]

8. Pi, Y.; Wang, X. Trajectory tracking control of a 6-DOF hydraulic parallel robot manipulator with uncertain load disturbances. Control Eng. Pract. 2011, 19, 185-193. [CrossRef]

9. Huang, S.N.; Kok, K.T.; Tong, H.L. Adaptive motion control using neural network approximations. Automatica 2002, 38, 227-233. [CrossRef]

10. Anderson, B.D.O.; Dehghani, A. Challenges of adaptive control-past, permanent and future. Annu. Rev. Control 2008, 32, 123-135. [CrossRef]

11. Roy, S.; Roy, S.B.; Kar, I.N. A New Design Methodology of Adaptive Sliding Mode Control for a Class of Nonlinear Systems with State Dependent Uncertainty Bound. In Proceedings of the 15th International Workshop on Variable Structure Systems (VSS), Graz, Austria, 9-11 July 2018; pp. 414-419.

12. Roy, S.; Kar, I.N. Adaptive sliding mode control of a class of nonlinear systems with artificial delay. J. Franklin Inst. 2017, 354, 8156-8179. [CrossRef]

13. Roy, S.; Kar, I.N. Adaptive-robust control of uncertain Euler-Lagrange systems with past data: A time-delayed approach. In Proceedings of the IEEE International Conference on Robotics and Automation, Stockholm, Sweden, 16-21 May 2016; pp. 5715-5720.

14. Roy, S.; Kar, I.N.; Lee, J.; Jin, M. Adaptive-Robust Time-Delay Control for a Class of Uncertain Euler-Lagrange Systems. IEEE Trans. Ind. Electron. 2017, 64, 7109-7119. [CrossRef]

15. Lee, M.C.; Aoshima, N. Identification and its evaluation of the system with a nonlinear element by signal compression method. Trans. SICE 1989, 25, 729-736. [CrossRef]

16. Slotine, J.-J.E.; Li, W. Applied Nonlinear Control; Prentice-Hall: Englewood Cliffs, NJ, USA, 1991.

17. Utkin, V.; Guldner, J.; Shi, J. Sliding Mode Control in Electro-Mechanical Systems; CRC Press: Boca Raton, FL, USA, 2009.

18. Wang, F.; Chen, Y. Dynamic characteristics of pressure compensator in underwater hydraulic system. IEEE/ASME Trans. Mechatron. 2014, 19, 777-787. [CrossRef]

19. Cao, Y.; Chen, X. Disturbance-observer-based sliding-mode control for a 3-DOF nanopositioning stage. IEEE/ASME Trans. Mechatron. 2014, 19, 924-931. [CrossRef]

20. Elmali, H.; Olgac, N. Sliding mode control with perturbation estimation (SMCPE): a new approach. Int. J. Control 1992, 56, 923-941. [CrossRef]

21. Moura, J.T.; Elmali, H.; Olgac, N. Sliding Mode Control with Sliding Perturbation Observer. J. Dyn. Syst. Meas. Control 1997, 119, 657. [CrossRef]

22. Yoon, S.M.; Lee, M.C.; Kim, C.Y. Sliding perturbation observer based reaction force estimation method of surgical robot instrument for haptic realization. Int. J. Hum. Robot. 2015, 12, 1550013. [CrossRef]

23. Kang, J.S.; Lee, M.C.; Yoon, S.M. Bilateral control based rupture protection method in surgical robot using improved master device. Int. J. Control Autom. Syst. 2016, 14, 1073-1080. [CrossRef]

24. Yu, X.; Zhihong, M. Fast terminal sliding-mode control design for nonlinear dynamical systems. IEEE Trans. Circuits Syst. I. Fundam. Theory Appl. 2002, 49, 261-264.

25. Yu, X.; Zhihong, M. Multi-input uncertain linear systems with terminal sliding-mode control. Automatica 1998, 34, 389-392. [CrossRef]

26. Galicki, M. Finite-time control of robotic manipulators. Automatica 2015, 51, 49-54. [CrossRef]

27. Chen, M.; Wu, Q.-X.; Cui, R.-X. Terminal sliding mode tracking control for a class of SISO uncertain nonlinear systems. ISA Trans. 2013, 52, 198-206. [CrossRef]

28. Feng, Y.; Yu, X.; Man, Z. Non-singular terminal sliding mode control of rigid manipulators. Automatica 2002, 38, 2159-2167. [CrossRef]

29. Yu, S.; Yu, X.; Shirinzadeh, B.; Man, Z. Continuous finite-time control for robotic manipulators with terminal sliding mode. Automatica 2005, 41, 1957-1964. [CrossRef]

30. Han, S.I.; Lee, J. Finite-time sliding surface constrained control for a robot manipulator with an unknown deadzone and disturbance. ISA Trans. 2018, 65, 307-318. [CrossRef] 
31. Merritt, H. Hydraulic Control Systems; John Wiley \& Sons: Ney York, NY, USA, 1967.

32. Kallu, K.D.; Wang, J.; Min, C.L. Sensorless reaction force estimation of the end effector of a dual-arm robot manipulator using sliding mode control with a sliding perturbation observer. Int. J. Control Autom. Syst. 2018, 16, 1367-1378. [CrossRef]

(C) 2019 by the authors. Licensee MDPI, Basel, Switzerland. This article is an open access article distributed under the terms and conditions of the Creative Commons Attribution (CC BY) license (http://creativecommons.org/licenses/by/4.0/). 